\title{
Model for High-Gain Fiber Laser Arrays
}

\author{
Jeffrey L. Rogers, Slaven Peleš, and Kurt Wiesenfeld
}

\begin{abstract}
Recent experiments have shown that a small number of fiber lasers can spontaneously form coherent states when suitably coupled. The observed synchrony persisted for a long time without any active control. In this paper, we develop a dynamical model for fiber laser arrays that is valid in the high gain regime. In the limiting case of a single laser analysis and simulations are presented that agree with physical expectations. Using simulations to examine array behavior we report results that are in qualitative agreement with laboratory observations.
\end{abstract}

Index Terms-Coherence, coupled lasers, laser array, optical fibers, self-organization, synchronizations, two-way coupling.

\section{INTRODUCTION}

I N DIFFERENTIATING the coherence displayed by optical masers (lasers) from "classical coherent behavior" Max Garbuny wrote "the synchronism which is a condition of coherence also extends to the timing of electronic interactions... This, in broad outline, is the nature of coherent interactions" [1]. The resulting macroscopic coherence from single lasers has led to a host of applications. Yet there are situations where an array of mutually coherent lasers offers distinct advantages. One notable example is the high-power optical source. The power output from a single laser is constrained by its physical characteristics. Common constraints include heat dissipation, mechanical breakdown, and power conversion efficiency. In contrast, relatively low-power fiber lasers can have excellent heat dissipation properties, relatively wide stability range, and greater than $70 \%$ pump conversion efficiency. An array of these lasers provides an attractive alternative to the single monolithic source.

To take full advantage of coherence array components must maintain zero relative phases. The far field spatial power distribution depends crucially on two aspects of the array electric fields: 1) the degree of coherence of the fields produced by distinct array elements and 2) the relative phases of these fields at the array output. In this paper, we call two lasers coherent if their respective electric fields maintain a constant relative phase. ${ }^{1}$ If this phase is zero the lasers are said to be in an inphase state; if the relative phase is $\pi$ the lasers are described as in an antiphase state. Due to inevitable variations between individuals, an array of $N$ isolated lasers will be incoherent and the resulting spatial intensity distribution is simply the sum of the intensity patterns

Manuscript received October 24, 2004; revised January 24, 2005. This work was supported in part by the Defense Advanced Research Projects Agency Contract MDA972-02-C-0050.

J. L. Rogers is with the Theoretical and Computational Physics Department, HRL Laboratories, LLC, Malibu, CA 90265 USA (e-mail: jeff@ @rl.com).

S. Peles and K. Wiesenfeld are with the School of Physics, Georgia Institute of Technology, Atlanta, GA 30332 USA.

Digital Object Identifier 10.1109/JQE.2005.847545

${ }^{1}$ Weaker definitions of coherence are possible, and may be relevant depending on circumstances. from each laser. Since adding the incoherent fields is equivalent to a random walk the peak intensity will increase as $N$. In contrast, if all $N$ lasers are inphase the output intensity is concentrated in a narrow pencil at broadside (zero angle). This state is particularly attractive since its peak intensity grows as $N^{2}$.

For these reasons a longstanding goal of optics research has been to produce laser arrays that are stable in the inphase state. While these investigations have produced a number of approaches and interesting results, the ultimate goal of designing inphase arrays in a manner that is scalable with laser number has remained elusive. A number of these approaches are variations of the master oscillator multiple amplifier (MOPA) concept. As the name implies MOPAs use an array of amplifiers to boost a master signal. Due to inevitable amplifier variations these arrays require active control of the relative phases through additional circuitry that limit both array stability and scalability. Recent MOPA architectures have utilized fiber laser developments to report output powers of $100 \mathrm{~W}$ [2]. Evolving this approach a step further, MOPA amplifiers have been replaced by lasers (oscillators) [3]. These injection locking approaches rely on slaving each individual array laser to the master source. A common implementation is arrays of evanescently coupled diode lasers. While diode arrays form coherent states, the antiphase state is most commonly observed [4]. Even if the inphase state can be formed in an array of diodes, the degree of control required to maintain this state as well as the basic physical architecture leaves the scalability to large $N$ of this solution an open and challenging question [5]-[7].

An alternative to these approaches is to use mutual synchronization to produce inphase arrays. Mutual synchronization has the advantage of being passive (no active control requirement). This approach also does not require a master source, rather it utilizes intrinsic nonlinearity and appropriate laser connectivity to facilitate a frequency and phase distribution that the array elements select. The phenomenon is familiar from nonlinear science studies in several areas of physics, biology, and engineering [8]-[10]: under the right circumstances a collection of coupled nonlinear oscillators will spontaneously form a synchronized dynamical state. At this level of description, the laser array dynamics is governed by a set of coupled nonlinear oscillator equations, either differential equations [11], [12] or iterative maps. Meanwhile, the detailed nature of the quantum mechanics of the inverted population and the electromagnetic cavity are not directly modeled. By focusing on the broader essentials of the problem we can investigate array sizes spanning several orders of magnitude and a wide range of coupling architectures in a reasonable amount of time.

A primary motivation for this paper is the recent experimental work on coupled fiber arrays at HRL Laboratories, LLC [13], [14]. These experiments were based on very general 

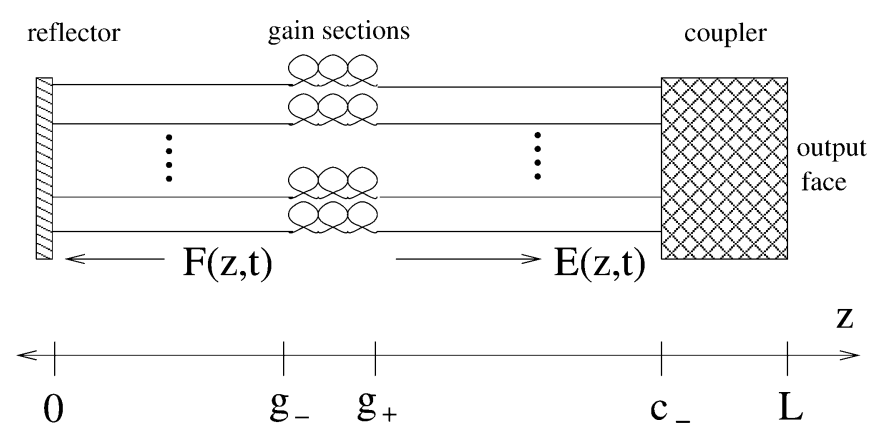

Fig. 1. Sketch of the laser array. Each component is described in the text (Sections II and III).

lessons from coupled oscillator and network studies. Here, we develop a more detailed theoretical description of the coupled laser system, keeping as general as possible the overall array architecture. A limitation worth noting is that the array configurations we study are composed of distinct lasers that interact over a relatively small cavity length. We note that interesting coherence has also been reported recently [15], [16] for a fiber system of multiple cores in a common glass cladding with interactions all along the cavity. The model we derive is applicable to lasers with both high and low gain. In Section II we derive a set of coupled nonlinear iterative maps for the system. The general formulation allows for a variety of distinct coupling schemes (Section III). In Section IV we examine the model analytically and numerically in the limiting case of a single laser; identifying three distinct types of behavior that reproduce qualitatively those seen in experiments. Finally, in Section V, we present some preliminary numerical results for the array.

\section{DESCRIPTION OF THE System}

We consider a set of $N$ fiber lasers as sketched in Fig. 1. On one end $(z=0)$ each separate fiber has a $100 \%$ reflective mirror. In the experiments at HRL Laboratories, LLC fiber gratings with better than $98 \%$ reflectivity were used. At the other cavity end the output face mirror has reflectivity $r$ which in practice is rather small. The lasers are independent except over the region labeled coupler $\left(c_{-} \leq z \leq L\right)$, where the fields mix. This coupling region may take on several forms in the laboratory. In between the mirrors are gain sections $\left(g_{-} \leq z \leq g_{+}\right)$that in practice may use various gain media. The gain sections are connected to the cavity ends by nongain, no-loss fiber sections.

In what follows, we calculate the change in the field amplitudes over one cavity round-trip. In this way we are led quite naturally to a set of coupled iterative maps that govern the dynamics of the electric fields. We then deduce the iterative maps that describe the evolution of the gains.

\section{A. Evolution of the Electric Fields}

We decompose the electric fields into counter-propagating waves. Let $E_{n}(z, t)$ be the complex phasor amplitude for the right-going wave in the $n$th fiber and let $F_{n}(z, t)$ be the corresponding quantity for the left-going wave. A cavity round-trip will begin with a right-going wave just prior to the output face at $z=L$. The round-trip is then made of the following steps where the waves: 1) bounce off of the partially reflecting output face; 2 ) propagate through the coupler (during this part the amplitudes mix); 3) freely propagate to the edge of the gain regions; 4) are amplified in passing through the gain regions; 5) freely propagate to the reflective mirrors; 6) reflect back; 7) propagate to the edge of the gain regions; 8) are amplified again; 9) freely propagate to the coupler; and finally, 10) pass through the coupler to the output face.

We now consider each of these steps in turn. At the coupler output face a fraction of each field is instantaneously reflected by a factor $0<r<1$ and with a $\pi$ phase shift, producing left propagating waves

$$
F_{n}(L, t)=-r E_{n}(L, t)
$$

Next, as $F_{n}$ passes through the coupler each wave is coupled to other members of the array. Upon emerging at time $t_{1}$

$$
\begin{aligned}
F_{n}\left(c_{-}, t_{1}\right) & =\sum_{m=1}^{N} S_{n m} F_{m}(L, t) \\
& =-r \sum_{m=1}^{N} S_{n m} E_{m}(L, t)
\end{aligned}
$$

where the matrix element $S_{n m}$ is the amount of the $m$ th field that scatters into the $n$th fiber. (In the limit of no coupling, $S$ is the identity matrix.)

As the waves propagate freely from the coupler to the edge of the gain sections at $z=g_{+}$, each picks up a phase factor that may vary. Let $t_{2, n}$ denote the time at which the $n$th wave reaches the gain section. Then the acquired phase shift is $\psi_{n}=$ $\omega_{c}\left(t_{2, n}-t_{1}\right)$, where $\omega_{c}$ is the carrier frequency, and

$$
\begin{aligned}
F_{n}\left(g_{+}, t_{2, n}\right) & =e^{j \psi_{n}} F_{n}\left(c_{-}, t_{1}\right) \\
& =-r e^{j \psi_{n}} \sum_{m=1}^{N} S_{n m} E_{m}(L, t) .
\end{aligned}
$$

The net effect is that each row of the coupling matrix is multiplied by a phase shift. The same is true for the phase shifts picked up within the gain sections and during the free propagation from the gain sections to the reflectors. In order to keep our notation as simple as possible, we shall combine all of these contributions into the cumulative phase shifts $\phi_{n} / 2$.

Passing through the gain sections and to the gratings at $z=0$ produces

$$
F_{n}\left(0, t_{3, n}\right)=-r e^{G_{n} / 2} e^{j \phi_{n} / 2} \sum_{m=1}^{N} S_{n m} E_{m}(L, t)
$$

Next, upon reflection off the mirrors at $z=0$, each left-going wave picks up a $\pi$ phase shift and becomes a right-going wave $E_{n}\left(0, t_{3, n}\right)=-F_{n}\left(0, t_{3, n}\right)$, assuming perfect reflectivity. Finally, in propagating back to the starting point the gain, coupler, and free-propagation phase shift contributions are the same as before, so that

$$
E_{n}\left(L, t+T_{n}\right)=\sum_{m=1}^{N} S_{n m} e^{G_{m} / 2} e^{j \phi_{m} / 2} E_{m}\left(0, t_{3, m}\right)
$$


where $T_{n}$ is the total round-trip time. Putting this all together, we obtain the following discrete map to describe the dynamics of the electromagnetic fields

$$
E_{n}\left(L, t+T_{n}\right)=r \sum_{\ell=1}^{N} S_{n \ell} e^{G_{\ell}+j \phi_{\ell}} \sum_{m=1}^{N} S_{\ell m} E_{m}(L, t) .
$$

At this point, a common step is to assume that the field amplitudes vary only a little over one round-trip, which allows one to recast the discrete map as a differential equation. We will not make this slowly varying wave approximation: because of the low reflectivity of the output mirror we are especially interested in the high-gain limit, and in this limit the changes in the field can be substantial.

\section{B. Evolution of the Gain Fields}

We now turn to the gain variables $G_{n}$. The counter propagating intensities are

$$
\begin{aligned}
& I_{n}^{+}(z, t)=\left|E_{n}(z, t)\right|^{2} \\
& I_{n}^{-}(z, t)=\left|F_{n}(z, t)\right|^{2} .
\end{aligned}
$$

Assuming, as before, perfect reflectivity at $z=0$ implies

$$
I_{n}^{+}(0, t)=I_{n}^{-}(0, t) .
$$

We use the Rigrod analysis [17] for two-way fibers, which is based on two partial differential equations for intensities and one equation for the time evolution of the atomic gain

$$
\begin{aligned}
\frac{\partial I_{n}^{+}(z, t)}{\partial z}= & +N_{n}(z, t) \sigma I_{n}^{+}(z, t) \\
\frac{\partial I_{n}^{-}(z, t)}{\partial z}= & -N_{n}(z, t) \sigma I_{n}^{-}(z, t) \\
\frac{\partial N_{n}(z, t)}{\partial t}= & R_{n}^{p}(z, t)-\frac{N_{n}(z, t)}{\tau} \\
& -\frac{\sigma}{\hbar \omega} N_{n}(z, t)\left[I_{n}^{+}(z, t)+I_{n}^{-}(z, t)\right] .
\end{aligned}
$$

In these equations $N_{n}(z, t)$ is the number of inverted atoms per unit length distributed along the $n$th fiber, $\sigma$ is the stimulated emission cross section, $\tau$ is the relaxation time, and $R_{n}^{p}$ is the pumping rate of the $n$th fiber. In the Rigrod analysis time derivatives in (8) and (9) are assumed small and ignored in comparison to the exponential spatial dependence of the gains.

We can use these equations to deduce the temporal evolution of the gain fields by integrating $N_{n}(z, t)$ over the fiber length $z$

$$
\hat{N}_{n}(t)=\int_{0}^{L} N_{n}(z, t) d z .
$$

Substituting (8) and (9) into (10) produces

$$
\begin{aligned}
\frac{\partial N_{n}(z, t)}{\partial t}=R_{n}^{p}(z, t)-\frac{N_{n}(z, t)}{\tau} & \\
& -\frac{1}{\hbar \omega}\left[\frac{\partial I_{n}^{+}(z, t)}{\partial z}-\frac{\partial I_{n}^{-}(z, t)}{\partial z}\right] .
\end{aligned}
$$

Upon integrating over the fiber length $z \in[0, L]$ this becomes

$$
\begin{aligned}
\frac{d \hat{N}_{n}}{d t}= & \hat{R}_{n}^{p}(t)-\frac{\hat{N}_{n}(t)}{\tau} \\
& -\frac{1}{\hbar \omega}\left[I_{n}^{+}(L, t)-I_{n}^{+}(0, t)-I_{n}^{-}(L, t)+I_{n}^{-}(0, t)\right]
\end{aligned}
$$

where the total pumping rate $\hat{R}_{n}^{p}(t)$ is defined by

$$
\hat{R}_{n}^{p}(t)=\int_{0}^{L} R_{n}^{p}(z, t) d z
$$

Using (7) to eliminate the intensities at the gratings $(z=0)$ in (12) yields

$$
\frac{d \hat{N}_{n}(t)}{d t}=\hat{R}_{n}^{p}(t)-\frac{\hat{N}_{n}(t)}{\tau}+\frac{1}{\hbar \omega}\left[I_{n}^{-}(L, t)-I_{n}^{+}(L, t)\right] .
$$

The next step is to eliminate $I_{n}^{-}$in favor of $I_{n}^{+}$. To do this, first integrate (9) over the fiber length $z \in[0, L]$ to get

$$
\ln \left[\frac{I_{n}^{-}(L, t)}{I_{n}^{-}(0, t)}\right]=-\sigma \hat{N}_{n}(t)
$$

Similarly, (8) leads to

$$
\ln \left[\frac{I_{n}^{+}(L, t)}{I_{n}^{+}(0, t)}\right]=\sigma \hat{N}_{n}(t)
$$

Subtracting (15) from (14) gives

$$
\ln \left[\frac{I_{n}^{-}(L, t) I_{n}^{+}(0, t)}{I_{n}^{+}(L, t) I_{n}^{-}(0, t)}\right]=-2 \sigma \hat{N}_{n}(t) .
$$

Using (7) to cancel the intensities at the gratings and exponentiating the result produces

$$
I_{n}^{-}(L, t)=e^{-2 \sigma \hat{N}_{n}(t)} I_{n}^{+}(L, t) .
$$

Finally, by defining the gain fields as

$$
G_{n}(t)=2 \sigma \hat{N}_{n}(t)
$$

and using (16) to eliminate $I_{n}^{-}(L, t)$ from (13) we find the governing differential equations for the gains

$$
\frac{d G_{n}}{d t}=\frac{1}{\tau}\left[G_{n}^{p}(t)-G_{n}(t)\right]+\frac{2}{\tau I_{\mathrm{sat}}}\left(e^{-G_{n}(t)}-1\right)\left|E_{n}(t)\right|^{2}
$$

where $n=1,2, \ldots, N$ and we have introduced

$$
\begin{aligned}
G_{n}^{p}(t) & =2 \sigma \tau \hat{R}_{n}^{p}(t) \\
I_{\text {sat }} & =\frac{\hbar \omega}{\sigma \tau} .
\end{aligned}
$$

\section{Final Iterated Map Model}

Together (6) and (18) provide a quantitative description for the dynamics of the laser array. However, based on the type of 
experiments we have in mind a few simplifying assumptions can be made.

First, we assume that all round-trip times $T_{n}$ are the same. This is a reasonable since in the laboratory fibers are several meters long and they can be cut accurately enough so that all round-trip times are the same to within a negligible error. On the other hand, one must be careful about the effect of this approximation on the phase shifts $\phi_{n}$ in (6): A miniscule change in fiber length, say less than $1 \mu \mathrm{m}$, is still of the order of the carrier wavelength, and therefore can introduce a phase shift of order of $\pi$. For this reason, we set $T_{n}=T$ for all $n$, but retain the corresponding phase factors $e^{j \phi_{n}}$. We also note that changes in the fiber lengths as large as the carrier wavelength may also occur due to the Kerr effect, temperature change or stress. Hence, the phase shifts $\phi_{n}$ can be well represented by some random distribution of angles.

The evolution equation for the electric fields (6) then becomes

$$
E_{n}(t+T)=r \sum_{\ell=1}^{N} S_{n \ell} e^{G_{\ell}(t)+j \phi_{\ell}} \sum_{m=1}^{N} S_{\ell m} E_{m}(t) .
$$

Rather than deal with a hybrid dynamical system which involves the iterated map (19) and the differential equation (18), we reduce the latter to an iterated map. Since $G_{n}$ varies slowly, we can assume it is constant over intervals comparable to the round-trip time $T$. This is also true for the pumping $G_{n}^{p}$. If we integrate (18) over time $T$ we obtain

$$
\begin{aligned}
G_{n}(t+T)=G_{n}(t)+ & \frac{T}{\tau}\left[G_{n}^{p}-G_{n}(t)\right] \\
& -\frac{2 T}{\tau I_{\text {sat }}}\left(1-e^{-G_{n}(t)}\right)\left\langle I_{n}(t)\right\rangle
\end{aligned}
$$

where

$$
\left\langle I_{n}(t)\right\rangle=\frac{1}{T} \int_{t}^{t+T} d t\left|E_{n}(t)\right|^{2}
$$

is a time average of the field intensity in the fiber $n$ over one round-trip. In what follows we use a somewhat cruder approximation and take $\left\langle I_{n}(t)\right\rangle \approx I_{n}(t)$. Substituting this into (20) yields the final equations of motion

$$
\begin{aligned}
E_{n}(t+T)= & \sum_{m=1}^{N} A_{n m}(t) E_{m}(t) \\
G_{n}(t+T)= & G_{n}(t)+\epsilon\left[G_{n}^{p}-G_{n}(t)\right] \\
& -\frac{2 \epsilon}{I_{\text {sat }}}\left(1-e^{-G_{n}(t)}\right) I_{n}(t)
\end{aligned}
$$

where $A_{n m}=r \sum_{\ell} S_{n \ell} e^{G_{\ell}+j \phi_{\ell}} S_{\ell m}$ and $\epsilon=T / \tau$. The coefficients $A_{n m}$ have only implicit time dependence. The fluorescence time $\tau$ is usually much longer than the round-trip time $T$, so the parameter $\epsilon$ is a small number. For the fiber lasers under consideration $\epsilon \sim 10^{-4}$.

\section{COUPLing}

An essential component of the array architecture is the coupling region, where fields from the individual array fibers interact. While evanescent coupling between electric fields is used to motivate our formulation the results are sufficiently general that they can be used to describe a variety of coupling schemes. We will limit our discussion to single mode lasers.

Fiber crosstalk [18] is an effect caused by evanescent coupling between electric fields that allows light to leak back and forth between fibers. Over a small increment of coupler length $d z$ the electric fields will satisfy [19]

$$
\frac{d \mathbf{E}}{d z}=j M \mathbf{E} \text {. }
$$

The electric fields of the individual fibers are the components of the $N$-element vector $\mathbf{E}=\left(E_{1}, E_{2}, \ldots, E_{N}\right)$, while $M$ is an $N \times N$ matrix whose terms are the pair interactions over $d z$. The terms in $M$ are equivalent to overlap integrals for electric field envelopes between pairs of array components over small lossless coupler displacements. Since the surrounding glass is assumed to be isotropic the integrals must be equivalent under symmetric permutation. Thereby the matrix $M$ is real and symmetric. In principle, the elements of $M$ can be calculated from the physical properties of the coupler.

Equation (24) is a linear matrix equation and can be solved analytically. Along the coupler of length $L^{\prime}$ the solution is

$$
\mathbf{E}\left(L^{\prime}\right)=T e^{j \int_{0}^{L^{\prime}} d z M(z)} \mathbf{E}(0)
$$

where $T$ denotes a time ordering operator and the integration interval $\left[0, L^{\prime}\right]$ is equal to $\left[c_{-}, L\right]$ in Fig. 1 . To evaluate the propagator (coupling matrix) $S=T \exp \left[j \int_{0}^{L^{\prime}} d z M(z)\right]$ we use a generalization of Euler's formula to find

$$
S=\lim _{n \rightarrow \infty} \prod_{m=n}^{1}\left[1+j \frac{L^{\prime}}{n} M\left(\frac{m}{n} L^{\prime}\right)\right] .
$$

If the entries of $M$ do not depend on $z$, the coupling matrix $S$ reduces to

$$
S=\lim _{n \rightarrow \infty}\left(1+j \frac{L^{\prime}}{n} M\right)^{n}=e^{j L M} .
$$

In general, the coupling matrix $S$ will have complex elements that must satisfy the additional constraints imposed by the assumptions that are made (e.g., no-loss). Characterization of couplers in the laboratory is complicated by the need to measure coupling phases as well as coupling intensities.

\section{SINGLE FIBER DYNAMICS}

To assist in understanding the iterative map model defined by (22) and (23) we first consider the case of a single laser $(N=1)$. Setting $A(t)=r e^{G(t)}$, where $r$ is the field reflection coefficient at the output face, (22) implies

$$
E(t+T)=r e^{G(t)} E(t) .
$$

Assuming $G(t)$ is real, we obtain a map for the intensity

$$
I^{\prime}=r^{2} e^{2 G} I
$$

Our notation drops explicit reference to time and the prime denotes a temporal $T$-shift (i.e., one iteration of the map or cavity round-trip). From the gain equation (23), we have

$$
G^{\prime}=G+\epsilon\left[G^{p}-G-2\left(1-e^{-G}\right) I\right] .
$$


The single laser map admits two fixed point solutions

$$
\tilde{I}=0, \quad \tilde{G}=G^{p}
$$

and

$$
\tilde{I}=\frac{G^{p}-\ln \left(\frac{1}{r}\right)}{2(1-r)}, \quad \tilde{G}=\ln \left(\frac{1}{r}\right) .
$$

The eigenvalues of the Jacobian matrix for the map evaluated at the fixed point given by (31) are $\lambda_{1}=r^{2} e^{2 G^{p}}$ and $\lambda_{2}=1-\epsilon$. Since, by definition, $0<\epsilon<1$ this fixed point is stable for as long as

$$
G^{p}<\ln \left(\frac{1}{r}\right)
$$

Physically, there is no lasing in this pumping regime, as the loss due to partial reflection is larger than the gain induced by pumping.

For the other fixed point (32) the eigenvalues of the Jacobian matrix are

$$
\lambda_{1,2}=1-\frac{\epsilon}{2}(1+2 r \tilde{I}) \pm \sqrt{\frac{\epsilon^{2}}{4}(1+2 r \tilde{I})^{2}-4 \epsilon(1-r) \tilde{I}}
$$

In the case of real eigenvalues, they will both be smaller than one as long as $\tilde{I}>0$, or expressed in terms of pumping parameter

$$
G^{p}>\ln \left(\frac{1}{r}\right) \text {. }
$$

Light intensity is of course always larger than zero, but sometimes it is convenient to treat $\tilde{I}$ as a parameter instead of the pumping $G^{p}$ and let it assume both negative and positive values. From expressions (33) and (35) we conclude there is a (transcritical) bifurcation at $G^{p}=\ln (1 / r)$. This corresponds to the onset of lasing, as the gain due to pumping becomes larger than the loss through the output face.

What is left to show is that the eigenvalues (34) are indeed real in a finite neighborhood around $\tilde{I}=0$. To do so we express the discriminant $D(\tilde{I})=\left(\epsilon^{2} / 4\right)(1+2 r \tilde{I})^{2}-4 \epsilon(1-r) \tilde{I}$ as a function of $\tilde{I}$. This is negative inside an interval $\left(I_{1}, I_{2}\right)$, where

$$
I_{1,2}=\frac{4(1-r)-\epsilon r \pm \sqrt{[4(1-r)-\epsilon r]^{2}-\epsilon^{2} r^{2}}}{2 \epsilon r^{2}}
$$

are solutions of equation $D(\tilde{I})=0$. A direct consequence is that both $I_{1,2}>0$ as long as the parameters $r, \epsilon<1$. In Fig. 2 we plot a few hundred iterations after an initial transient over the range of $G^{P}$ values shown. The reflection coefficient is chosen to be $r=0.187$. Simulations demonstrate that the fixed point solution persists over a range of parameter values and the onset of lasing occurs at $G^{p}=1.67664 \ldots$, in agreement with the prediction of (35).

In the case when (34) are complex we find that the square magnitude of the eigenvalues for the "on" state is

$$
\left|\lambda_{1,2}\right|^{2}=1-\epsilon(1+6 r \tilde{I}-4 \tilde{I})
$$

which is smaller than one as long as

$$
\tilde{I}<I_{h}=\frac{1}{4-6 r} .
$$

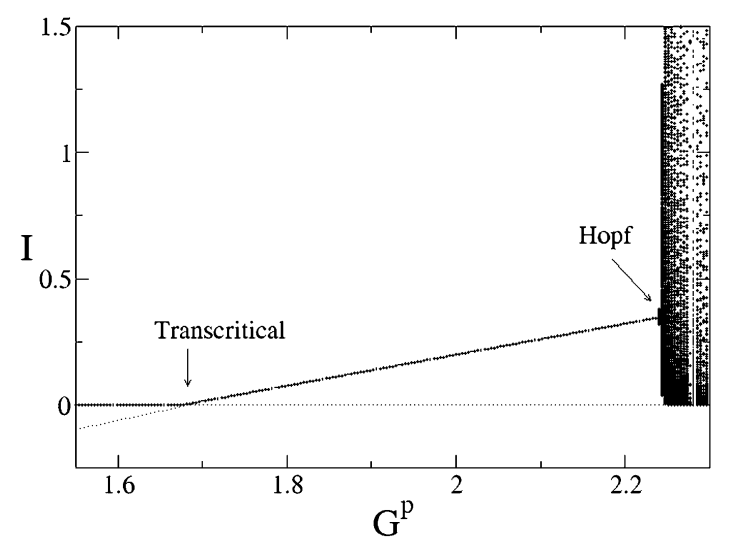

Fig. 2. Plots of a few hundred intensity iterations after transients have decayed over a range of pumping values. At the transcritical bifurcation, the laser turns on and the output intensity is a constant that increases linearly with the pumping until the Hopf bifurcation. At larger pumping values, the laser displays complex temporal dynamics as well as additional dynamical instabilities.

At $\tilde{I}=I_{h}$ the system undergoes a Hopf bifurcation (see Figs. 2 and 3). Expressed in terms of the pumping parameter, the Hopf bifurcation occurs at

$$
G^{p}=\ln \left(\frac{1}{r}\right)+\frac{1-r}{2-3 r}
$$

As the pumping is increased beyond this value the fixed point (32) becomes unstable, and a stable limit cycle is created. The system behaves as a relaxation oscillator and instead of a constant intensity field, there is a series of pulses at the output face (Fig. 4).

In order for a Hopf bifurcation to occur, $I_{1,2}$ have to be real, with $I_{h} \in\left(I_{1}, I_{2}\right)$. For the range of parameters under consideration $\left(r \sim 10^{-1}, \epsilon \sim 10^{-4}\right)$ this is always true, although it is possible to think about a laser model where the Hopf bifurcation will be absent.

With further increase of the pumping parameter the system undergoes another dynamical transition. The bifurcation diagram in Fig. 3 reveals very rich dynamics beyond this point. There are a number of frequency locking intervals as well as indications of intermittent chaos.

Note that because we use discrete time, the field takes on only a finite number of values in the frequency locked regime. These are evident as "windows" in the bifurcation diagram Fig. 3(b). Regardless of whether the gain field oscillations are frequency locked with the round-trip time, the pulses appear to be quite regular for this range of pumping parameter (Fig. 4).

It is interesting to note that the onset of both the transcritical and Hopf bifurcations are independent of $\epsilon$, the ratio between fluorescence time and the round-trip time. However, our simulations indicate that the onset of the subsequent stability transition is proportional to $\epsilon$. In the bifurcation graph in Fig. 3 we used an exaggerated value $\epsilon=0.01$ for better clarity of presentation. For the time scales found in experiments $\left(\epsilon \sim 10^{-4}\right)$ our model suggests that this transition will occur after a very small increment of pumping parameter $G^{p}$ with respect to the Hopf onset, given by (39). 

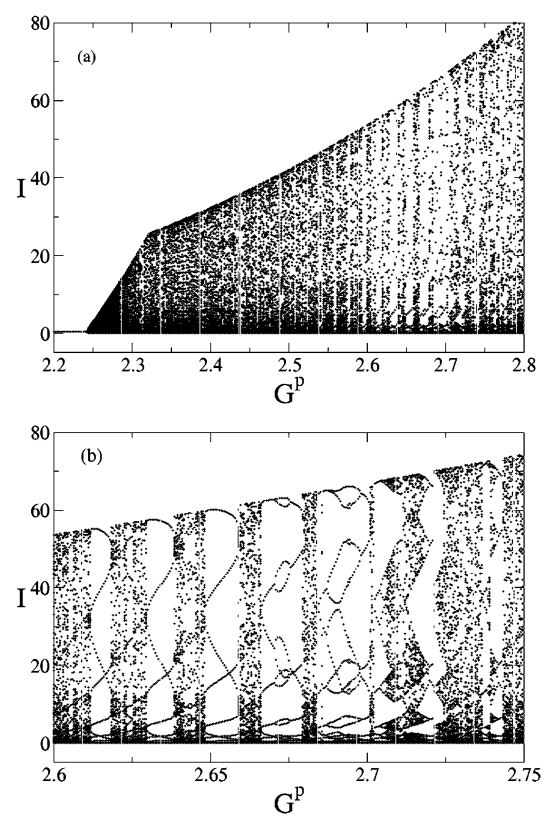

Fig. 3. Extension to large pumping and intensities of the single laser bifurcation diagram shown in Fig. 2. The Hopf bifurcation and subsequent stability transition are shown in (a) with an enlarged segment of the diagram that reveals frequency lockings between oscillations of the field and round-trip times shown in (b).
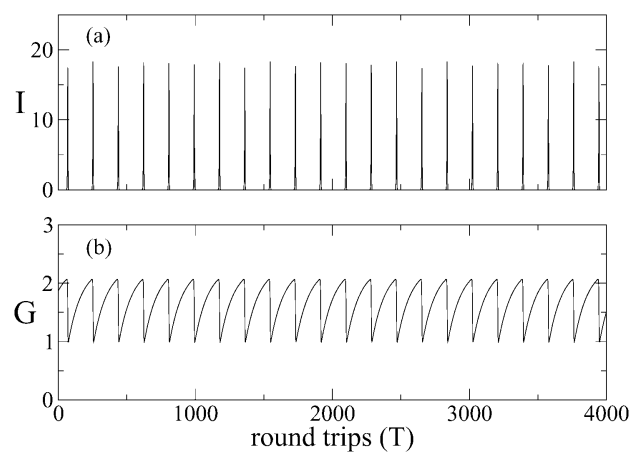

Fig. 4. (a) Single laser electric field intensity and (b) gain field during 4000 cavity round-trips. At this pumping of $G^{p}=2.3$ the model is in a pulsing state.

\section{LASER ARRAY SiMULATIONS}

Simulations of the array provide evidence of mutual synchronization producing array coherence. The experiments reported in demonstrated that groups of lasers can form coherent states without the need for any active control. The first of these experiments used 5 lasers that were effectively all-to-all coupled. To model this system we numerically solved (22) and (23) with $N=5$ and an all-to-all coupling matrix. Based on an analogy with fiber crosstalk we approximate the diagonal terms of the coupling generator to be $M_{n n}=2.546$ and the off diagonal terms to be 0.046 . We also assume that all phase factors in (19) are $e^{j \phi_{n}}=1$. Simulations begin with each laser in an off-state: the initial electric fields are chosen at random so that the magnitudes are of order 0.1 and the phases are uniformly distributed. To cut down on transients the initial gain fields are set to have a magnitude less then, but of the same order as, the pumping. All of the lasers are assumed to have the same constant pumping value of $G^{P}$. The model is iterated until steady state behavior is sustained, as defined by stable moments for the intensity of
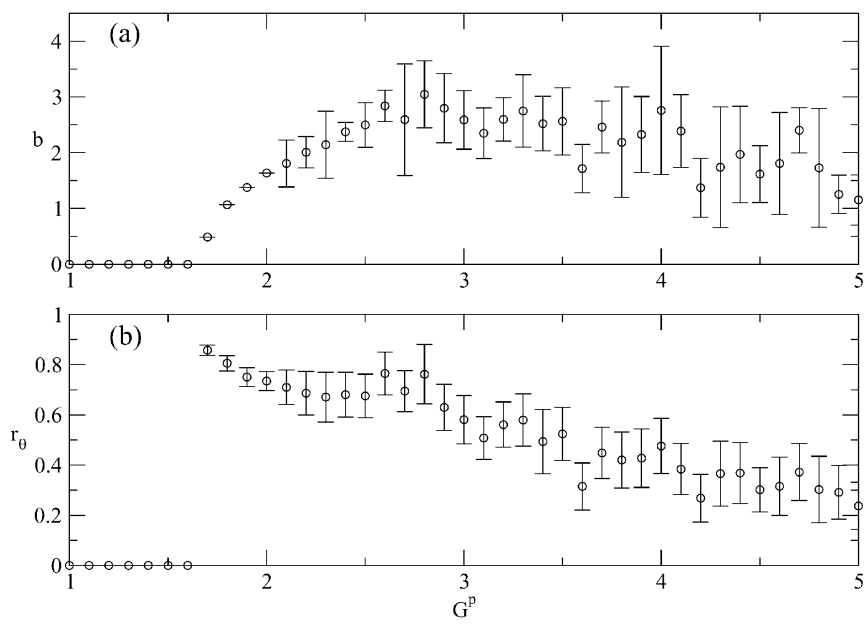

Fig. 5. (a) Brightness measure and (b) the phase order parameter over a range of pumping values for an array of five $(N=5)$ all-to-all coupled lasers.

each laser. The electric fields and gain fields are then recorded for a sufficient additional time to establish the long term array behavior. Typical array behaviors were found to be independent of initial conditions.

To better describe the array states we introduce two related order measures. Motivated by the array brightness the first is defined as

$$
b e^{j \psi}=\sum_{m=1}^{N} E_{m}
$$

and quantifies the degree of electric field amplitude and phase coherence. Physically, the array brightness is directly proportional to the magnitude $b$. The second measure is an order parameter describing electric field phase order defined as

$$
r_{\theta} e^{j \xi}=\frac{1}{N} \sum_{m=1}^{N} e^{j \theta_{m}}
$$

where $\theta_{m}$ is the $m$ th electric field phase. If all these phases are the same, the magnitude $r_{\theta}=1$. In contrast, if the phases are uniformly distributed, $r_{\theta}=0$. Due to the initial conditions of these simulations the order parameter magnitudes are initially small, with $r_{\theta} \approx 0.1$.

Our simulations demonstrate that the single laser dynamics provide substantial insight into the array behavior. For one, the onset of lasing occurs at the same value of the pump parameter. This is indicated by the phase order parameter (Fig. 5) which assumes around $80 \%$ of its maximum value at the onset of lasing and remains so for $G^{p}<3$. (The phase order parameter is not defined when the fields are zero, but the natural convention is to set it to zero in this regime.) For larger values of pumping the coherence gradually decays and for $G^{p}>5$ the phases are approximately randomly distributed. Transient response of the system is of order $10^{5}$ round-trip times (Fig. 6). This corresponds to approximately a few milliseconds in the laboratory .

Output intensity of a single fiber increases with the pump. In order to obtain large intensity at the array output face it is necessary to maintain coherence as the pumping is increased. By calculating brightness of the array we find that largest output intensity is reached for pumping values $2.5<G^{p}<3$. For a number of the coupling matrices we investigated, like the one 

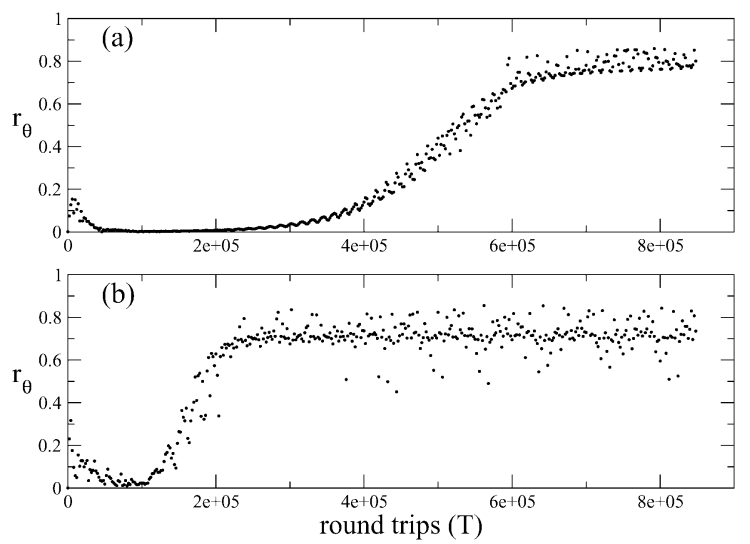

Fig. 6. Temporal evolution of the phase order parameter for pumping (a) $G^{P}=1.8$ and (b) $G^{P}=2.1$.

used for the simulations summarized in Fig. 5, the array phase coherence decayed with increasing pumping. It was found that the rate and degree of this decay depended sensitively on the coupling matrix. In the 5-to- 1 experiments shown in the array selected a nearly ideal inphase state when operated around twice the onset pumping. With the properly chosen coupling matrix the model introduced here will display nearly inphase states over a range of pumping values.

Summarizing, we have described a theoretical model for coupled laser arrays with high gain. Using a somewhat idealized all-to-all coupling between fibers we observed the spontaneous mutual synchronization similar to the laboratory investigations that motivated this work. Of course, no physically realizable coupler will have the perfect permutation symmetry we assumed. On the other hand we can view the five different pumping parameters to be independent and tunable control parameters. In the cases shown we set them all equal. It is reasonable to expect that for couplers with different (lower) symmetry it would be possible to find a range of pumping parameters that would result in a spontaneous synchronization.

\section{ACKNOWLEDGMENT}

The authors would like to thank M. Minden, C. Jones, M. Mangir, and especially $\mathrm{H}$. Brusselbach for their collaboration over the course of this project. They would also like to thank S. Wandzura for helpful discussions and A. Siegman for guidance in the early stages of the theory.

\section{REFERENCES}

[1] M. Garbuny, Optical Physics. New York: Academic, 1965, pp. 304-304.

[2] A. Liem, H. Z. J. Limpert, and A. Tünnermann, "100-w single-frequency master-oscillator fiber power amplifier," Opt. Lett., vol. 28, no. 17, pp. 1539-1539, Sep. 2003.

[3] C. Wang, "Master and slave oscillator array system for very large multiline lasers," Appl. Opt., vol. 17, no. 1, pp. 83-86, Jan. 1978.

[4] A. Khibnik, Y. Braiman, T. Kennedy, and K. Wiesenfeld, "Phase model analysis of two lasers with injected field," Physica D, vol. 111, pp. 295-310, 1998.

[5] D. Botez and L. Mawst, "Phase-locked laser arrays revisited," IEEE Circuits Devices Mag., vol. 12, no. 6, pp. 25-31, Nov. 1996

[6] Y. Liu, H. Liu, and Y. Braiman, "Injection locking of individual broadarea lasers in an integrated high-power diode array," Appl. Phys. Lett., vol. 81, no. 6, pp. 978-980, Aug. 2002.
[7] - "Simultaneous injection locking of couples of high-power broadarea lasers driven by a common current source," Appl. Opt., vol. 41, no. 24, pp. 5036-5039, Aug. 2002.

[8] I. I. Blekhman, Synchronization in Science and Technology. New York: ASME, 1988.

[9] S. H. Strogatz, Sync: The Emerging Science of Spontaneous Order. New York: Hyperion, 2003.

[10] A. Pikovsky, M. Rosenblum, and J. Kurths, Synchronization-A Universal Concept in Nonlinear Sciences. Cambridge, UK: Cambridge Univ. Press, 2001.

[11] S. Wang and H. Winful, "Dynamics of phase-locked semiconductor laser arrays," Appl. Phys. Lett., vol. 52, pp. 1774-1776, May 1988.

[12] H. Winful and S. Wang, "Stability of phase locking in coupled semiconductor laser arrays," Appl. Phys. Lett., vol. 53, pp. 1894-1896, Jan. 1988.

[13] J. L. Rogers, M. Minden, and H. Brusselbach, "Self-organized inphase laser array," presented at the 7th Experimental Chaos Conf., San Diego, CA, Aug. 2002.

[14] M. Minden, H. W. Bruesselbach, J. L. Rogers, M. S. Mangir, D. C. Jones, G. J. D. Dunning, L. Hammon, A. J. Solis, and L. Vaughan, "Self-organized coherence in fiber laser array," in Proc. SPIE_Fiber Lasers: Technology, Systems, and Applications, vol. 5335, Jun. 2004, pp. 89-97.

[15] P. K. Cheo, A. Liu, and G. G. King, "A high-brightness laser beam from a phase-locked multicore yb-doped fiber laser array," IEEE Photon. Technol. Lett., vol. 13, no. 5, pp. 439-441, May 2001.

[16] E. J. Bochove, P. K. Cheo, and G. G. King, "Self-organization in a multicore fiber laser array," Opt. Lett., vol. 28, no. 14, pp. 1200-1202, Jul. 2003.

[17] A. E. Siegman, Lasers. Mill Valley, CA: University Science Books, 1986.

[18] A. W. Snyder and J. D. Love, Optical Waveguide Theory. New York: Chapman and Hall, 1983.

[19] W.-P. Huang, "Coupled-mode theory for optical waveguides: an overview," J. Opt. Soc. Amer. A, vol. 11, no. 3, Mar. 1993.

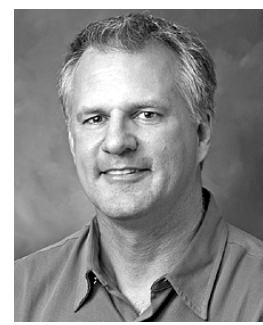

Jeffrey L. Rogers received the B.S. and M.S. degrees in physics from the Florida Atlantic University, Boca Raton, FL, in 1992 and 1994, respectively, the M.S. degree in physics from Emory University, Atlanta, GA, in 1996, and the Ph.D. degree in physics from Georgia Institute of Technology, Atlanta, GA, in 2001.

He is currently a Research Scientist in the Theoretical and Computational Physics Department at HRL Laboratories, LLC, Malibu, CA, where he is responsible for applying nonlinear sciences to a range of applications, including optical- and millimeter-wave systems.

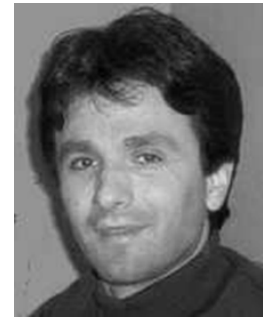

Slaven Peleš was born in Sarajevo, Bosnia and Herzegovina, in 1968. He received the Diploma in physics from University of Sarajevo, Sarajevo, Bosnia and Herzegovina, and the Ph.D. degree in physics from the University of Manitoba, Winnipeg, MB, Canada.

Currently, he is a Joseph Ford Fellow in the School of Physics, Georgia Institute of Technology, Atlanta, GA. His research interests are in dynamical systems and synchronization phenomena.

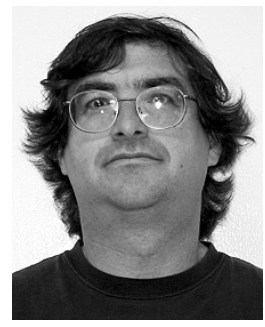

Kurt Wiesenfeld received the B.S. degree in physics from the Massachusetts Institute of Technology, Cambridge, MA, in 1979 and the Ph.D. degree in physics from the University of California (UC) at Berkeley, in 1985.

After postdoctoral positions at UC Santa Cruz and Brookhaven National Laboratory, Upton, NY, he joined the physics faculty at Georgia Institute of Technology, Atlanta, GA, in 1987.

Dr. Wiesenfeld is a Member of the Society of Inthe American Physical Society. dustrial and Applied Mathematics, and a Fellow of 